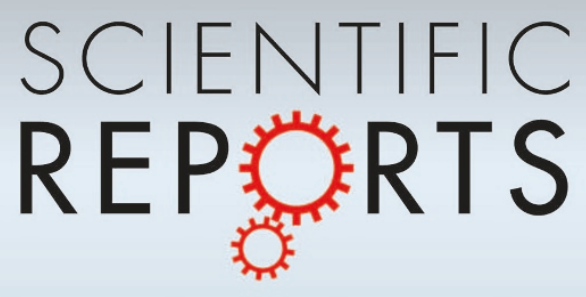

OPEN

SUBJECT AREAS:

CHEMICAL

ENGINEERING

ELECTRONIC PROPERTIES AND

DEVICES

NANOWIRES

SENSORS AND BIOSENSORS

Received

1 August 2014

Accepted

22 December 2014

Published

20 January 2015

Correspondence and requests for materials should be addressed to J.J. (jsjang@plaza.snu. ac.kr)

* These authors contributed equally to this work.

\section{Highly Sensitive and Multifunctional} Tactile Sensor Using Free-standing $\mathrm{ZnO} /$
PVDF Thin Film with Graphene Electrodes
for Pressure and Temperature Monitoring

\author{
James S. Lee ${ }^{*}$, Keun-Young Shin'*, Oug Jae Cheong', Jae Hyun Kim² \& Jyongsik Jang'
}

\begin{abstract}
'World Class University program of Chemical Convergence for Energy \& Environment, School of Chemical and Biological Engineering, Seoul National University, 151-742, Korea, ${ }^{2}$ Manufacturing Technology Team, Infra Technology Service Center, Device Business, Samsung Electronics, San \# 16 Banwol-Dong, Hwasung-City, Gyeonggi-Do, Korea.
\end{abstract}

We demonstrate an $80-\mu \mathrm{m}$-thick film (which is around $15 \%$ of the thickness of the human epidermis), which is a highly sensitive hybrid functional gauge sensor, and was fabricated from poly(vinylidene fluoride) (PVDF) and $\mathrm{ZnO}$ nanostructures with graphene electrodes. Using this film, we were able to simultaneously measure pressure and temperature in real time. The pressure was monitored from the change in the electrical resistance via the piezoresistance of the material, and the temperature was inferred based on the recovery time of the signal. Our thin film system enabled us to detect changes in pressure as small as $10 \mathrm{~Pa}$ which is pressure detection limit was $10^{3}$-fold lower than the minimum level required for artificial skin, and to detect temperatures in the range $20-120^{\circ} \mathrm{C}$.

r. lectronic skin (e-skin) mimics some of the functionality of human skin, aiming to provide sensory responses to mechanical, thermal, chemical, biological and optical stimuli. Therefore, multifunctional electronic sensors are of great interest for medical and industrial applications. In particular, thin-film systems have been investigated for use in sensor devices, due to the convenient, low-cost processing techniques and flexibility. Moreover, the development of a flexible thin-film device that can replace the epidermis has important applications in the treatment of injuries. Much effort has been devoted to enhancing the performance of pressure-sensing films. Integrated, strain-gauge sensors have been reported by Kahp-Yang Shu et al., ${ }^{1}$ that could detect pressure, shear and torsion. In contrast, relatively little attention has been paid to sensing of combined external stimuli in a single device, such as sensing mechanical strain and temperature. It is not straightforward to design a system that can discriminate among different stimuli, because the fabrication of such multifunctional sensors typically involves integration of a number of organic and inorganic devices using hybrid matrix arrays and circuit elements, which involves complex, expensive processes. ${ }^{2-13}$ Thus, it is still challenging to realize a sensor that enable to detect multiple stimuli accurately and simultaneously.

To date, much effort has been devoted to investigating the piezoelectric and pyroelectric properties of thin films for fabrication of multifunctional sensors. The piezoelectric and pyroelectric properties of thin films enable fabrication of multifunctional sensors. Reverse-piezoelectricity can respond to an applied mechanical displacement by inducing an electric potential and pyroelectricity. ${ }^{14-19}$ An example of such a material is poly(vinylidene fluoride) (PVDF), which has attracted much interest as a next-generation piezoelectric and pyroelectric material because of its light weight, flexibility, low power consumption, and non-toxicity. ${ }^{20-24}$ We have previously demonstrated the piezoelectric effect of PVDF for application as an acoustic acuator. ${ }^{4}$ The enhanced permittivity, which is related to the polarization and dipole moment of PVDF, is key factor for improving the piezoelectric and pyroelectric properties of PVDF. For this reason, the semiconductor zinc oxide $(\mathrm{ZnO})$ is of interest, as it may increase the piezoelectric response, is thermally stable, and may increase the permittivity of PVDF ${ }^{25,26}$. Therefore, a hybrid PVDF matrix with a $\mathrm{ZnO}$ nanofiller may enable fabrication of a hybrid piezoelectric/pyroelectric sensor.

Furthermore, piezoresistive and pyroresistive sensing, which transduces mechanical displacement and temperature signals into an electrical signal, may be a valuable and simple monitoring method for small structures ${ }^{1}$. In particular, a change in the electron mobility or resistance can be measured using a transistor structure. 
Here we report a highly sensitive hybrid functional gauge sensor using a PVDF thin film and vertically grown $\mathrm{ZnO}$ nanorods. As electrodes, a graphene was utilized which is derived from the vacuum-assisted reduction of graphene oxide (GO) film by inkjet printing. To our knowledge, this is the first experimental demonstration of the detection of two different independent stimuli (i.e., pressure and temperature) simultaneously and in real time. The pressure was inferred from the change in the electrical resistance via the piezoresistance of the material, and the temperature was determined based on the recovery time of the signal. The morphology of the $\mathrm{ZnO}$ nanostructures was controlled to maximize the response of the device, and the output signals were monitored while controlling the pressure and temperature to calibrate the device.

\section{Results}

Fabrication of a PVDF- and ZnO-based sensor. Fig. 1a and b show a schematic diagram and photograph of the flexible multilayer device. A large areal density of $\mathrm{ZnO}$ nanorods was embedded in the PVDF on a reduced graphene oxide (rGO)-treated flexible polyethylene terephthalate (PET) thin film. First, exfoliated GO aqueous ink was modified on the flexible PET substrate via inkjet printing, and reduced for use as an electrode ${ }^{27}$. The $\mathrm{ZnO}$ nanorods were then grown, followed by a hydrothermal treatment, as shown in Fig. 1a. The $20-w t \%$ PVDF solution was dissolved in a $1: 1$ mixture of acetone and dimethylformamide (DMF), and was spin-coated onto the $\mathrm{ZnO}$ nanorods, which then coagulated ${ }^{28}$. A poling process was then carried out under a strong constant electric field of $300 \mathrm{kV} / \mathrm{cm}$ at $90^{\circ} \mathrm{C}$ to induce piezoelectricity and pyroelectricity in the $\mathrm{PVDF}^{4}$. A

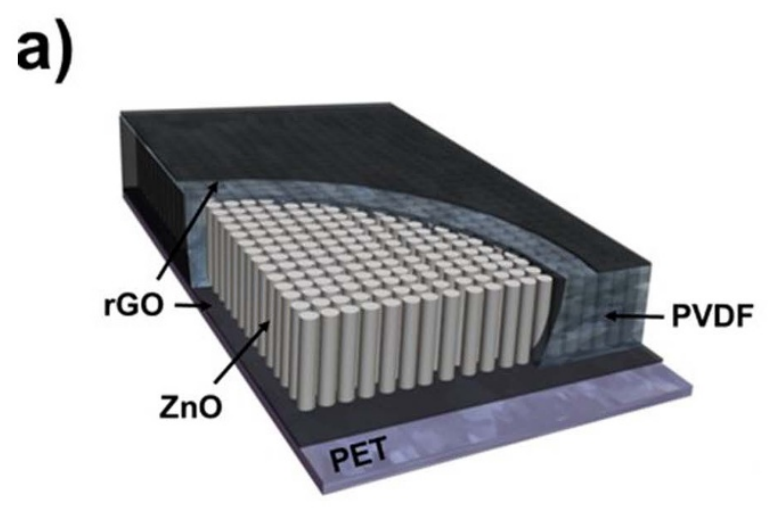

b)

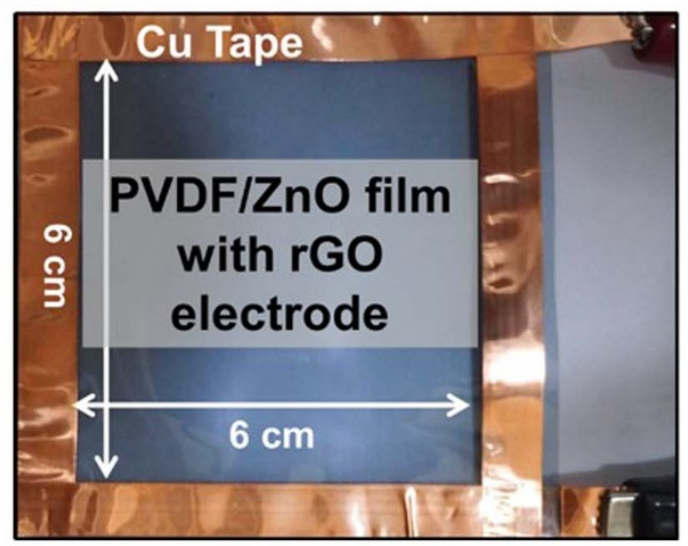

Figure $1 \mid$ (a) Schematic diagram and (b) a photograph of the device consisting of the $\mathrm{ZnO} / \mathrm{PVDF}$ composite film and rGO electrodes. top electrode of conductive ink was coated onto the PVDF/ZnO nanorod composite film using inkjet printing.

Characterization of the rGO electrode. To fabricate the conductive ink electrode, an aqueous solution GO was synthesized using the Hummers and Offeman method ${ }^{29-31}$. Fig. 2a shows an atomic force microscopy (AFM) image of the fabricated GO ink, which was a few microns thick, and was composed of 2-3-nm-thick bilayers, as well as some multilayers $(>3 \mathrm{~nm})$. To demonstrate the reduction of GO solution to graphene, X-ray photoelectron spectroscopy (XPS) and Raman spectroscopy analysis were carried out. Fig. 2b shows deconvoluted $\mathrm{C}$ 1s XPS spectra of the inkjet-printed GO and the reduced GO ( $\mathrm{rGO}$ ) sheets ${ }^{18,19}$. The $\mathrm{C} 1 \mathrm{~s}$ signal of $\mathrm{GO}$ has three main components: the $\mathrm{C}=\mathrm{C}$ and $\mathrm{C}-\mathrm{C}$ bond vibrations in the in aromatic rings $(285.0 \mathrm{eV}), \mathrm{C}-\mathrm{O}(286.5 \mathrm{eV})$ in epoxy or hydroxyl groups, and $\mathrm{C}=\mathrm{O}(288.5 \mathrm{eV})$ peaks originating from carbonyl and carboxy groups. The ratio $I_{C-O} / I_{C-C}$ decreased from 1.1440 (GO) to 0.2432 (rGO). In addition, a Raman spectrum of the printed rGO sheets on the substrate film is shown in Fig. 2c. The Raman spectra of rGO clearly exhibited the characteristic peaks of the $\mathrm{D}$ band $\left(\sim 1350 \mathrm{~cm}^{-1}\right)$, which indicate the typical defects ascribed to structural edge effects, such as epoxides covalently bonded to the base plane, the $G$ band $\left(\sim 1580 \mathrm{~cm}^{-1}\right)$, which indicates a graphite carbon structure, and the ration of $I_{D} / I_{G}$ was $0.77^{30,31}$.

Characterization of the $\mathrm{ZnO}$ nanostructures. The $\mathrm{ZnO}$ nanostructures were fabricated using a seed solution zinc acetate $\left(\mathrm{Zn}\left(\mathrm{CH}_{3} \mathrm{COO}\right)\right)_{2}$ dissolved in ethanol, which was spin-coated onto the rGO-treated PET film. The $\mathrm{ZnO}$ nanorods were grown from a mixture of zinc nitrate hexahydrate $\left(\mathrm{Zn}\left(\mathrm{NO}_{3}\right)_{2} \cdot 6 \mathrm{H}_{2} \mathrm{O}\right)$, hexamethylenetetramine and water at $90^{\circ} \mathrm{C}$. The morphology of the $\mathrm{ZnO}$ nanorods was controlled by varying the time and concentration of the solution of growth precursor. During the early stages of the hydrothermal method, $\mathrm{ZnO}$ nucleates spontaneously from the ionized $\mathrm{Zn}(\mathrm{OH})_{4}{ }^{2-}$ seed, to form a hexagonal nanostructure in the mixture, which then initiates the growth of one-dimensional (1D) nanorods by increasing the temperature and concentration. As shown in Figs. $3 a$ and b, fieldemission scanning electron microscopy (FE-SEM) images reveal that a high density of $\mathrm{ZnO}$ nanorods $\left(1.42 \times 10^{10} \mathrm{~cm}^{-2}\right)$ and disks were formed. Furthermore, high-resolution transmission electron microscopy (HR-TEM) images show that the $\mathrm{ZnO}$ nanorods and nanodisks had a well resolved single-crystalline wurtzite structure, with a lattice constant of $0.52 \mathrm{~nm}$ in the [0001] growth direction ${ }^{32,33}$. This indicates that they formed a uniform hexagonal crystal structural without crystalline defects (see inset Fig. $3 a$ and $b$ ). The length of the $\mathrm{ZnO}$ nanorods was $\sim 300 \mathrm{~nm}$, and the diameter was $\sim 85 \mathrm{~nm}$; the $\mathrm{ZnO}$ nanodisks were $\sim 30 \mathrm{~nm}$ in length and $\sim 100 \mathrm{~nm}$ in diameter. To investigate the crystal structure of the $\mathrm{ZnO}$ nanorods and nanodisks, X-ray diffraction (XRD) analysis was carried out, as shown in Fig. 3c. All of the diffraction peaks could be clearly indexed to the hexagonal $\mathrm{ZnO}$ with a lattice constant of $c=$ $0.5206 \mathrm{~nm}$, which is in good agreement with that of $\mathrm{ZnO}$ along the $c$-axis ${ }^{32}$.

Dielectric properties of the PVDF/ZnO device. The piezoelectric and pyroelectric properties of PVDF are strongly dependent on the $\beta$-phase content, owing to large net dipole moment, which originates from the all-trans structure ${ }^{4,34}$. Therefore, Fourier transform infrared (FTIR) spectroscopy was carried out to investigate the strong dielectric potential, based on the ratio of the $\alpha$ and $\beta$ phases $\left(I_{\beta} / I_{\alpha}\right)$, and the fraction of the $\beta$ phase, as shown in Fig. $4 \mathrm{a}$. Assuming that the IR absorption follows the Beer-Lambert law, $\beta$ content in the PVDF can be measured from:

$$
F(\beta)=\frac{X_{\beta}}{X_{\alpha}+X_{\beta}}=\frac{A_{\beta}}{\left(K_{\beta} / K_{\alpha}\right)+A_{\beta}}
$$


a)

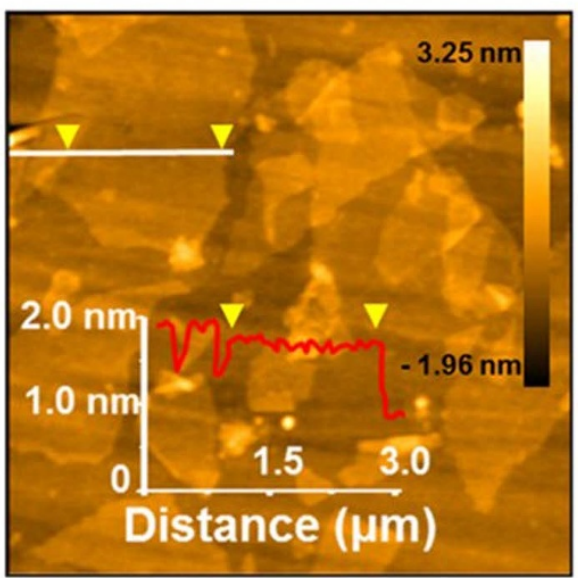

b)

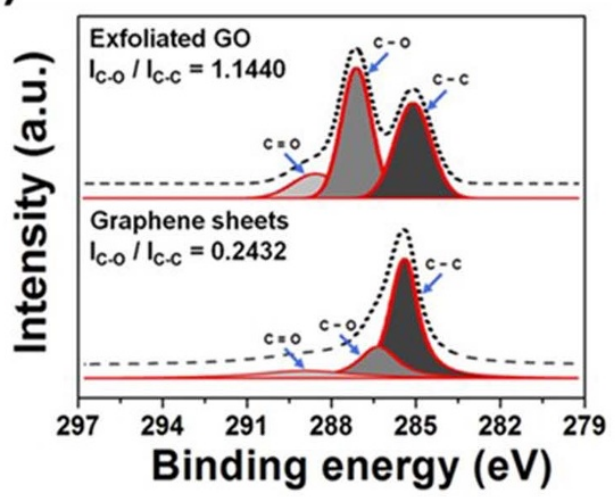

c)

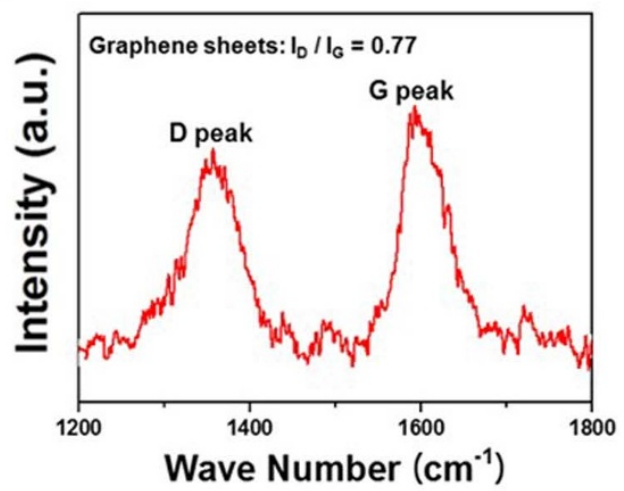

Figure $2 \mid$ (a) AFM image of the exfoliated GO solution. (b) XPS spectrum with fitted lines of the pristine and $\mathrm{rGO}$ solution for $\mathrm{C}-\mathrm{C}, \mathrm{C}-\mathrm{O}$ and $\mathrm{C}=\mathrm{O}$ bonds. (c) Raman spectra of the rGO electrodes.

where $A_{\alpha}$ and $A_{\beta}$ are the absorbance at 766 and $840 \mathrm{~cm}^{-1}$, respectively, and $K_{\alpha}=6.1 \times 10^{4} \mathrm{~cm}^{2} / \mathrm{mol}$ and $K_{\beta}=7.7 \times$ $10^{4} \mathrm{~cm}^{2} / \mathrm{mol}$ are the absorption coefficients of $\alpha$ and $\beta$ phases at the respective wavenumbers ${ }^{4}$. The PVDF thin film exhibited $I_{\beta} / I_{\alpha}$ $=2.33$, which corresponds to an $85 \% \beta$ phase. (For comparison, commercially available PVDF exhibited $I_{\beta} / I_{\alpha}=1.29$, which corresponds to a $73 \% \beta$ phase content.)

Generally, PVDF with a high $\beta$-phase content exhibits enhanced piezoelectric and pyroelectric responses, due to fact that the dipoles are aligned. Also, Fig. S1. shows that observed polarized optical microscopy morphology (POM) of the crystal growth of PVDF with a)

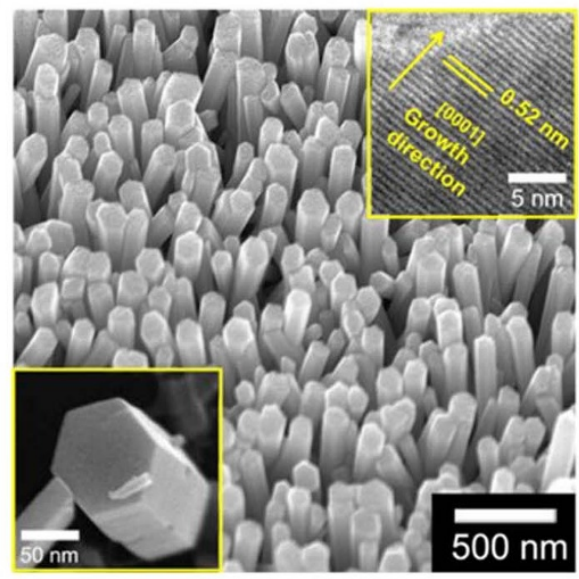

b)

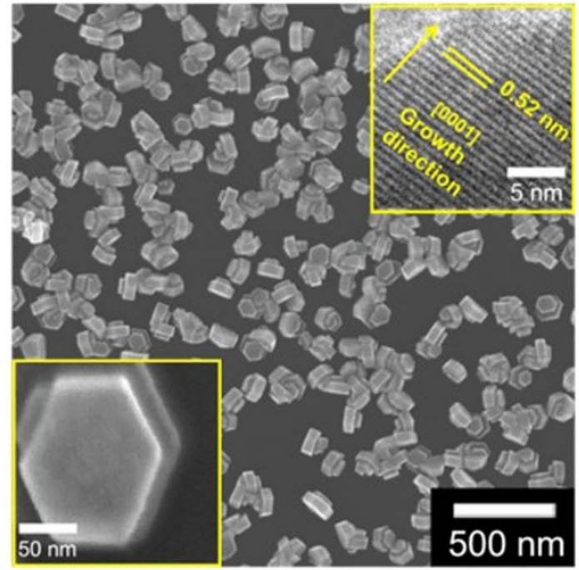

c)

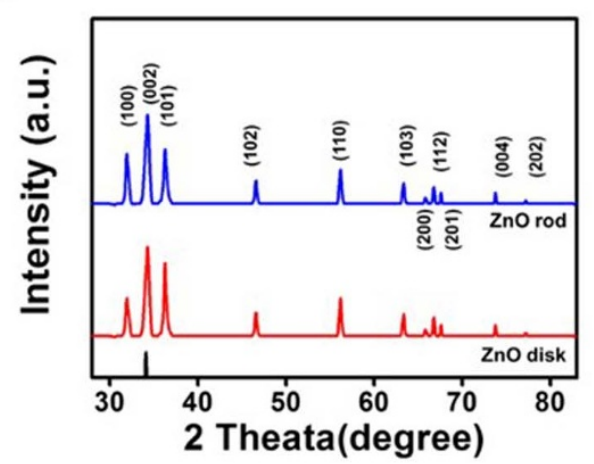

Figure 3 SEM images of (a) the $\mathrm{ZnO}$ nanorods and (b) the $\mathrm{ZnO}$ nanodisks. (c) XRD spectra to investigate the crystal structure of the $\mathrm{ZnO}$ nanorods and disks.

various $\mathrm{ZnO}$ fillers. All samples were isothermally crystallized at $170^{\circ} \mathrm{C}$ and maintained for $240 \mathrm{~s}$, and it cooled down to observe crystal growth morphology. During poling process of film, high external voltage was applied on the film and polarized $\mathrm{ZnO}$ induced $\beta$ phase of PVDF. Thus, regardless of $\mathrm{ZnO}$ morphology, it is clarified that $\mathrm{ZnO}$ acts as nuclei for PVDF crystallization. Therefore, we may expect favorable permittivity and losses of sensors fabricated using various $\mathrm{ZnO}$ and PVDF thin films. The dielectric properties of PVDF with the embedded $\mathrm{ZnO}$ nanorods can be estimated using the Havriliak-Negami and Fourier transfer relationship; i.e.: 
a)

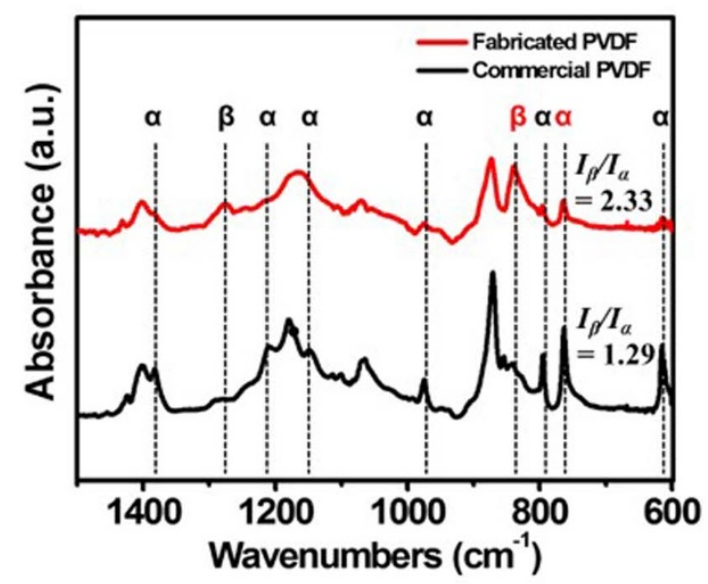

b)

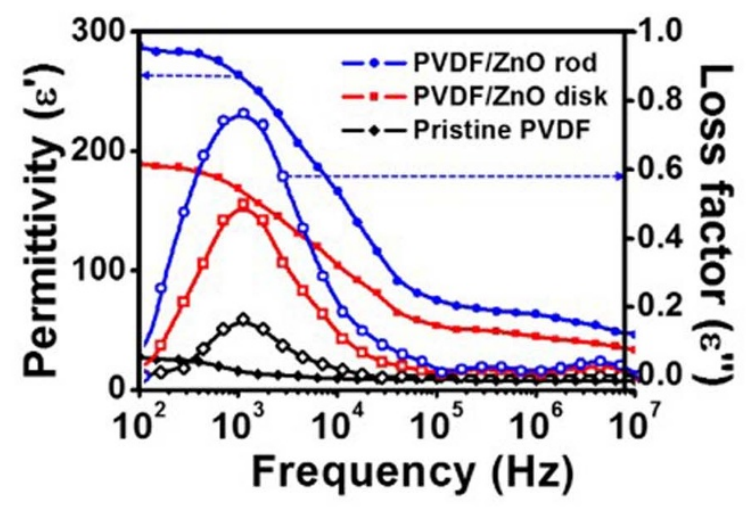

Figure $4 \mid$ (a) FTIR spectra of PVDF film fabricated as part of this work, and a commercially available PVDF film. The crystalline phases are indicated. (b) The permittivity and losses of the tactile sensor fabricated with PVDF and different $\mathrm{ZnO}$ nanostructures, as well as the PVDF thin film and the commercially available PVDF thin film.

$$
\varepsilon^{*}=\varepsilon^{\prime}+i \varepsilon^{\prime \prime}=\varepsilon_{\infty}+\frac{\Delta \varepsilon}{1+(i \omega \lambda)^{1-\alpha}}
$$

Where $\varepsilon^{\prime}$ is the dielectric constant, $\varepsilon^{\prime \prime}$ is the dielectric loss; the permittivity is therefore given by:

$$
\Delta \varepsilon=\varepsilon_{S}-\varepsilon_{\infty}
$$

where $\varepsilon_{s}$ is the static permittivity (i.e., when $\lim _{\omega \rightarrow 0} \varepsilon^{*}(\omega)$ ), and $\varepsilon_{\infty}$ is the high-frequency permittivity (i.e., when $\left.\lim _{\omega \rightarrow \infty} \varepsilon^{*}(\omega)\right)^{4,35,36}$.

The measured permittivities of the samples are listed in Fig. $4 \mathrm{~b}$. and Table S1. In general the permittivity of a dielectric is larger at lower frequencies than at higher frequencies, and embedding the $\mathrm{ZnO}$ in the PVDF significantly increased the dielectric constant. In particular, the $\mathrm{ZnO}$ nanorods possessed a greater dielectric constant than the $\mathrm{ZnO}$ disks, which is attributable to the larger aspect ratio of the $\mathrm{ZnO}$ nanorods, which resulted in a larger energy barrier.

The relaxation time $\lambda$ is related to the gradient of the loss factor that is associated with the interfacial polarization response of the dielectric material. The relaxation time is given by:

$$
\lambda=\frac{1}{2 \pi f_{\max }}
$$

where $f_{\text {max }}$ is the frequency of the peaks in the loss spectrum ${ }^{36}$. We find a relaxation time of $\lambda=159$ for the PVDF, $\lambda=130 \mu$ s for the $\mathrm{ZnO}$ nanodisks, and $\lambda=121 \mu$ s for the $\mathrm{ZnO}$ nanorods (see Table $\mathrm{S} 1$ ). Based on these dielectric parameters, the inclusion of $\mathrm{ZnO}$ in the PVDF film significantly increased the permittivity and reduced the polarization response time of the PVDF-based sensor devices. Since $\mathrm{ZnO}$ nanorods with PVDF possessed a higher dielectric constant, the concentration of seed solution was controlled to investigate effects of $\mathrm{ZnO}$ rods density. Approximately $30 \mathrm{wt} \%$ of zinc acetate dissolved seed solution grow $1.42 \times 10^{10} \mathrm{~cm}^{-2}$, and 25 and $35 \mathrm{wt} \%$ represent $0.68 \times 10^{10} \mathrm{~cm}^{-2}$ and $2.54 \times 10^{10} \mathrm{~cm}^{-2}$ of $\mathrm{ZnO}$ rods. As a result, $25 \mathrm{wt} \%$ of zinc acetate solution sparsely grow $\mathrm{ZnO}$ rods compare to $30 \mathrm{wt} \%$ of seed solution which result in reduced dielectric properties. Additionally, $35 \mathrm{wt} \%$ of seed solution limits dielectric properties of fabricated sample because exceeding density of $\mathrm{ZnO}$ rods caused aggregation between rods (Table S2).

Detecting pressure and temperature. To compare the pressuresensing properties of the PVDF and $\mathrm{ZnO} / \mathrm{PVDF}$ composite films, pressure stimuli were used, and the performance was measured from the change in the electrical resistance; i.e., $\Delta R=R_{\text {loading }}-R_{\text {Unloading }}$ (Fig. S2). The assembled device had an overlap area of $6 \times 6 \mathrm{~cm}^{2}$, and was measured with a gradual increase in pressure. Fig. 5a shows how the composite device formed of PVDF, $\mathrm{ZnO}$ nanorods and $\mathrm{ZnO}$ nanodisks responded to application of a constant pressure. The surface of compressed $\mathrm{ZnO}$ nanorods generates a negative potential, and the electrical contact with the rGO electrode layer and the forward bias of the Schottky barrier at the interface results in a change in the resistance of the device (Fig. S3). A difference in the response $\Delta R$ to the same pressure was observed between devices, and the PVDF/ZnO nanorod composite device exhibited the greatest signal in response to a given stimulus. Because of the $1 \mathrm{D}$ structure of the vertically grown $\mathrm{ZnO}$ nanorods, they generate an enhanced piezoelectric response to mechanical displacement than the $\mathrm{ZnO}$ nanodisks. The larger aspect ratio and permittivity lead to a change in the electrical resistance in response to even a slight deflection of the $\mathrm{ZnO}$ nanorods.

The pressure-sensing capability of the system was evaluated from the response $\Delta R$, as shown in Fig. $5 \mathrm{~b}$. The smallest detectable pressure using the $\mathrm{ZnO}$ nanorod/PVDF film was $10 \mathrm{~Pa}$ (giving $\Delta R=$ $0.062 \Omega$ ), which corresponds to $1 \mathrm{mg} \mathrm{mm}^{-2}$, which is 1000 -fold more sensitive than the minimum requirements for artificial $\operatorname{skin}^{3,37-40}$. The response times of the output signals from $\mathrm{ZnO}$ nanorod/PVDF device were obtained under various pressures at $20^{\circ} \mathrm{C}$. The recovery times exhibited similar as shown in Figs. $5 \mathrm{c}$ and $\mathrm{d}$. Additionally, three different conducting material of rGO, CNT and PEDOT: PSS were candidate to demonstrate pressure sensitivity as a function of various electrode (Fig. S4.). The sheet resistances of commercial poly(3,4-ethylenedioxythiophene):poly(styrenesulfonate) (PEDOT:PSS) (Sigma-Aldrich) and carbon nanotubes (CNT) (Sigma-Aldrich), and synthesized rGO were 34,10 and $0.7 \mathrm{k} \Omega / \mathrm{sq}$. As a result, $\mathrm{PVDF} / \mathrm{ZnO}$ rods with $\mathrm{rGO}$ electrode has highest sensitivity under constant pressure compare to other electrode utilized device.

To investigate the spatial resolution of the pressure-sensing ability, $\mathrm{ZnO} / \mathrm{PVDF}$ devices were fabricated, as shown in Fig. 6 . The film was divided into 144 regions, each $0.5 \times 0.5 \mathrm{~cm}^{2}$, and $\mathrm{Pt}$ weights were used to apply a pressure of $30 \mathrm{~Pa}$ at each division, one at a time. The output signal was measured from the change in the resistance using a pseudocolor plot, as shown in Fig. 6a. The two-dimensional (2D) intensity profiles of the $\mathrm{ZnO}$ nanorod/PVDF film are shown in Fig. $6 \mathrm{~b}$, and the $2 \mathrm{D}$ intensity profile of that formed using the $\mathrm{ZnO}$ nanodisk/PVDF film are shown in Fig. 6c. The distribution of the operational device area containing the $\mathrm{ZnO}$ nanorods in the PVDF thin film was $96 \%$, and that containing the $\mathrm{ZnO}$ nanodisks was $77 \%$. As the areal density of $\mathrm{ZnO}$ nanorods was larger, and significant 
a)

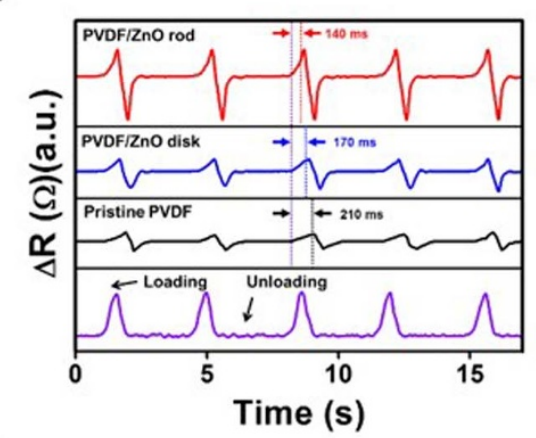

c)

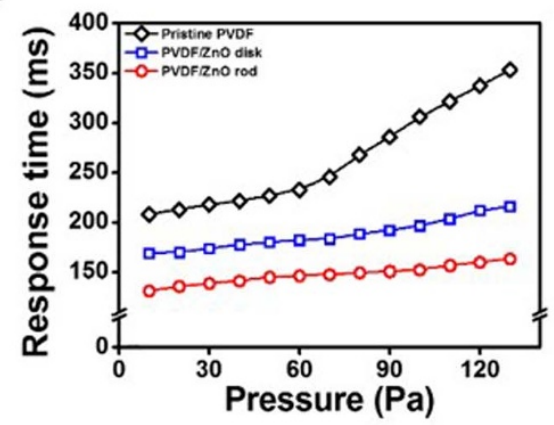

b)

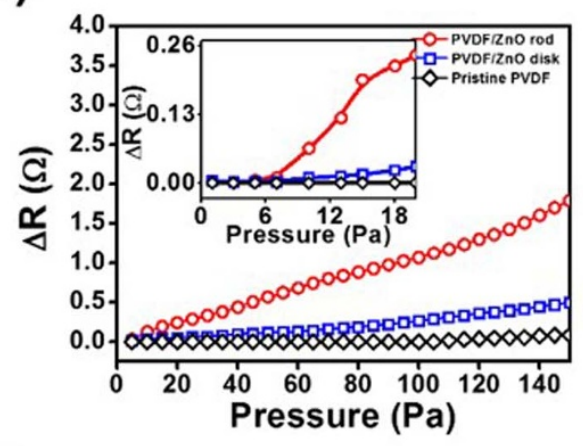

d)

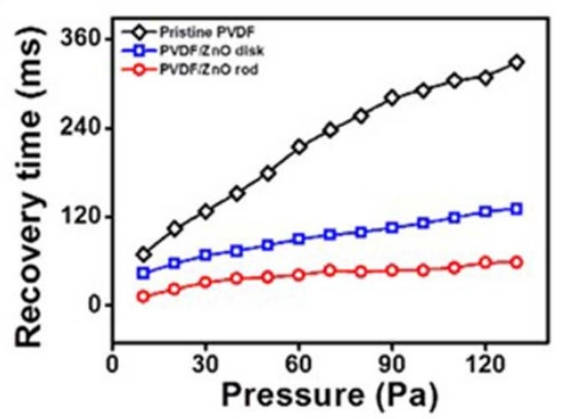

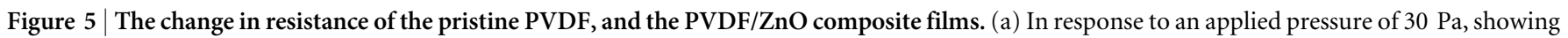
the response time. (b) The sensitivity of the films at $20^{\circ} \mathrm{C}$, using various Pt weights. (c) The response and (d) recovery times of the PVDF film, the $\mathrm{ZnO}$ nanodisk/PVDF film, and the $\mathrm{ZnO}$ nanorod/PVDF with various pressures.

aggregation did not occur, in contrast to the $\mathrm{ZnO}$ nanodisks, and so we may expect a more uniform pressure sensitivity with the $\mathrm{ZnO}$ nanorod/PVDF composite film.

The device composed of the $\mathrm{ZnO}$ nanorod/PVDF composite was used to measure the output signals with various temperatures and applied pressures. To evaluate the pyroelectric response, the $\mathrm{Pt}$ weight, which applied a pressure of $30 \mathrm{~Pa}$, were applied at 20, 70, and $120^{\circ} \mathrm{C}$ in the center of device, as shown in Fig. 7a. We found that the response was slower, and the recovery time longer, at higher temperatures. This is because the pyroelectricity originated from the PVDF, and the thermal energy released from the heated weight on the surface of PVDF thin film resulted in slower motion of the dipoles ${ }^{15,34,41-44}$. Loading at $20^{\circ} \mathrm{C}$ resulted in a recovery time of $190 \mathrm{~ms}$, loading at $70^{\circ} \mathrm{C}$ resulted in a recovery time of $680 \mathrm{~ms}$, and loading at $120^{\circ} \mathrm{C}$ in a recovery time of $1150 \mathrm{~ms}$.

As shown Figs. $7 \mathrm{~b}$ and $7 \mathrm{c}$, reproducible responses to changes in temperature and pressure were obtained. The independence of $\Delta R$ and the recovery time enabled measurement of pressure and temperature independently. Compare to obtained respons and recovery time, it is found that recovery time has wide delayed time range, $1150 \mathrm{~ms}$ at $120^{\circ} \mathrm{C}$ with $30 \mathrm{~Pa}$ whereas delayed response time observes $92 \mathrm{~ms}$. Additionally, the analyzed uniformity of temperature-sensing at the fabricated $\mathrm{ZnO}$ nanorods embedded PVDF thin film was $94 \%$ (Fig. S5). Considering these results, it is worth nothing that investigation of resistance difference make it feasible to measure the weight of pressure, and delayed recovery time play a pivotal role in determining the temperature of target object.

Application as tactile sensor. Mapping data of the two independent factors was evaluated based on external pressure loaded simultaneously and the temperature of the device. As shown in Figs. $8 \mathrm{a}$ and $8 \mathrm{~b}$, the applied pressure changed the resistance. Increasing the temperature of the object placed on the then film to apply the pressure increased the recovery time of the signal, and so the temperature of object could be calculated based on the recovery time. To investigate the scope for simultaneous pressure and temperature sensing in real time, a $15-\mu \mathrm{L}$ mineral oil droplet dropped from an initial height of $1.5 \mathrm{~cm}$ was measured under a low-noise environment, as shown in Fig. 8c. Data were collected during the initial impact, and during the time when the droplet rebounded from the surface, and impacted again, bouncing on the surface over a period of $1.2 \mathrm{~s}$, as shown in Fig. 8d. The maximum signal at $20^{\circ} \mathrm{C}$ following the impact of the droplet onto the film was $\Delta R=1.62 \Omega$, which is equivalent to a pressure of $90 \mathrm{~Pa}$; the recovery time was $180 \mathrm{~ms}$. The signal when a droplet with an unknown temperature was dropped from the same height was monitored. The maximum response was $\Delta R=1.61 \Omega$. However, recovery time was $815 \mathrm{~ms}$, which corresponds to a droplet temperature of $80^{\circ} \mathrm{C}$. The difference in the two peak shapes is attributable to the difference in the sample temperature of each droplet. The $20^{\circ} \mathrm{C}$ droplet that impacted on the film did not heat the film, because they were at an identical temperature. Therefore, the bouncing movement of a droplet can be detected after its first impact at $510 \mathrm{~ms}$. However, the $80^{\circ} \mathrm{C}$ droplet was able to transfer both kinetic energy and heat to the film. For this reason, the recovery time was prolonged. Therefore, this system enables independent measurement of pressure and temperature based on the magnitude of the change in the resistance and the recovery time of the signal.

\section{Discussion}

We have demonstrated a highly sensitive and multifunctional sensor using a PVDF/ZnO nanorod composite thin film. Existing approaches focus on improving the sensitivity, whereas here we could measure the pressure and temperature independently. The strategy used in the design and fabrication of $\mathrm{ZnO}$ nanorods embedded in the PVDF thin film represents a significant advance in the state of the art of nanotechnology fabrication methods. The high sensitivity to pressure and the ability to independently sense temper- 
a)

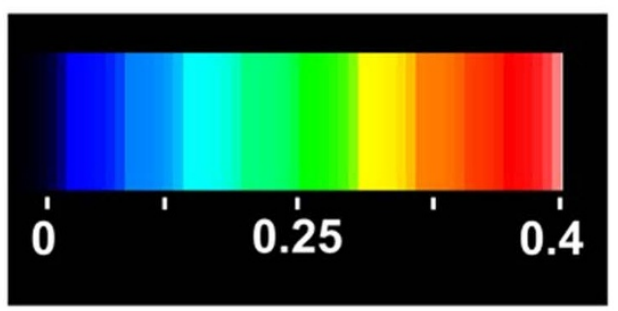

$\Delta R$ distribution $(\Omega)$

b)

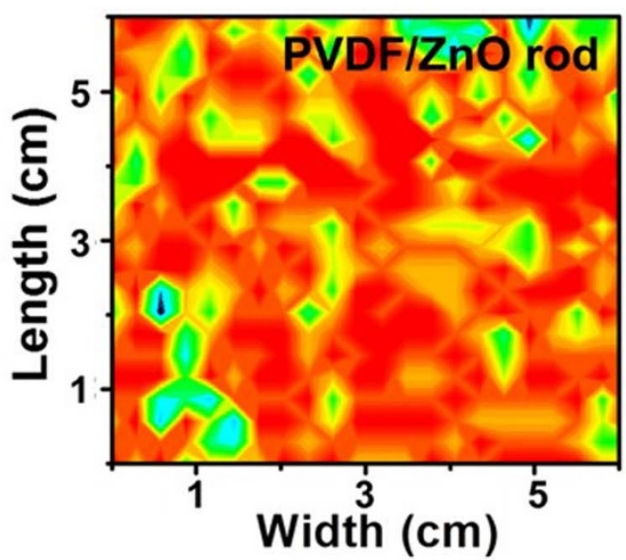

c)

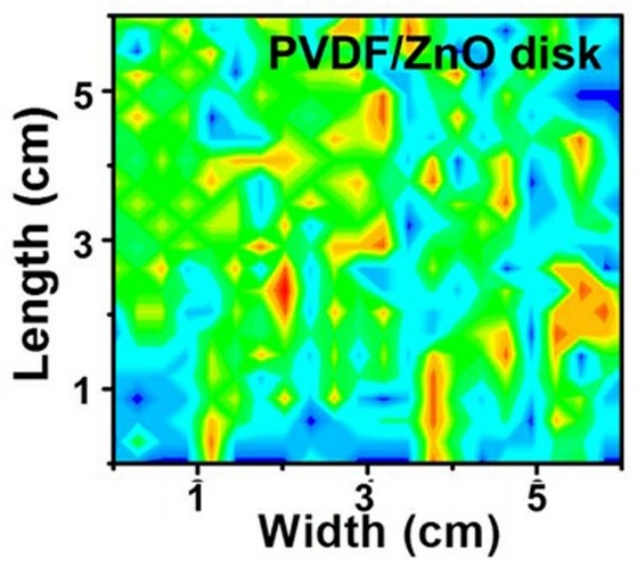

Figure $6 \mid$ (a) The spatial variation in the change in resistance. The effective area of fabricated device are $6 \times 6 \mathrm{~cm}^{2}$. (b) The $\mathrm{ZnO}$ nanorod/ PVDF film exhibited a higher pressure sensitivity than (c) the $\mathrm{ZnO}$ nanodisk/PVDF film. The film was divided into 144 regions $(0.5 \times$ $0.5 \mathrm{~cm}^{2}$ ) and $\mathrm{Pt}$ weights were used to apply a pressure of $30 \mathrm{~Pa}$ at each division, one at a time.

ature are attributable to the properties of the vertically grown $\mathrm{ZnO}$ nanorods and enhanced $\beta$-phase component of the PVDF film. The morphology of $\mathrm{ZnO}$ nanostructures was either disks or rods (Fig. 3), and could be controlled by varying the process conditions. As a result, the dielectric behavior of PVDF was improved owing to the piezoelectric barrier provided by the $\mathrm{ZnO}$ nanorods (Fig. 4). a)

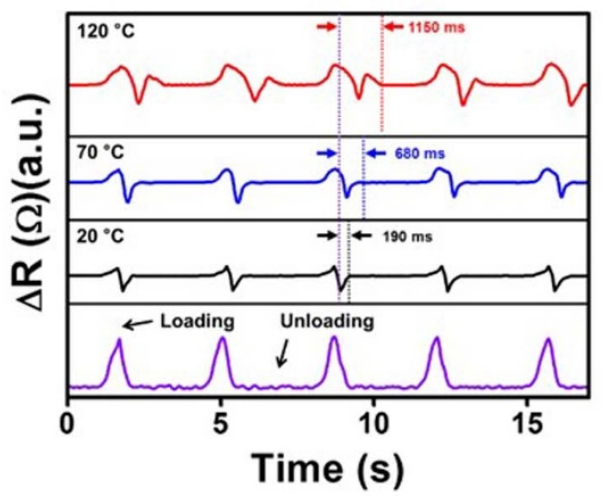

b)

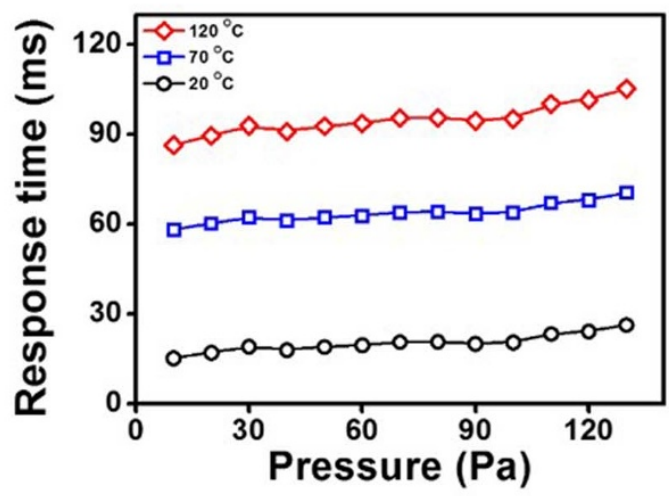

c)

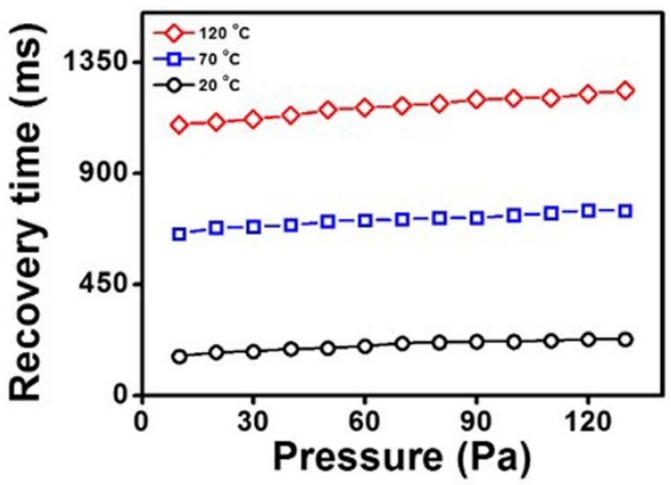

Figure $7 \mid$ (a) The output resistance of the PVDF/ZnO nanorod device at various temperatures, 20,70 , and $120^{\circ} \mathrm{C}$, under constant pressure of $30 \mathrm{~Pa}$ in the center of device. (b) The response to different pressures and (c) the recovery time at various temperatures.

The composite sensing film exhibited high sensitivity and accuracy, and a real-time response. Using the $\mathrm{ZnO}$ nanorods embedded in the PVDF thin film, the pressure sensor could respond to very small pressures, with a minimum detectable pressure of $10 \mathrm{~Pa}$. Moreover, we were able to detect temperatures in the range 20$120^{\circ} \mathrm{C}$. Pyroelectricity induced by the surface of the PVDF film resulted in variation in the output recovery time, from which we could infer the temperature of target object (see Fig. 8).

In conclusion, we fabricated composite $\mathrm{ZnO} / \mathrm{PVDF}$ thin films, and used these structures to independently measure the pressure applied to the film and the temperature of the object used to apply the pressure. The pressure detection limit was $10^{3}$-fold lower than the 
a)

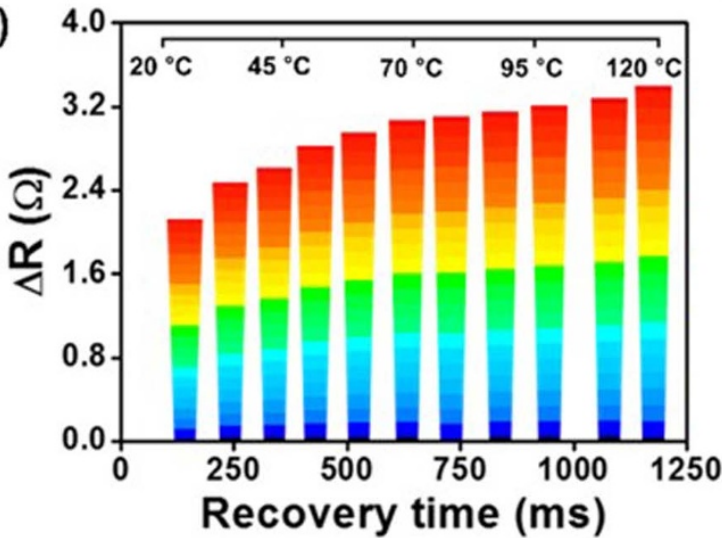

b)

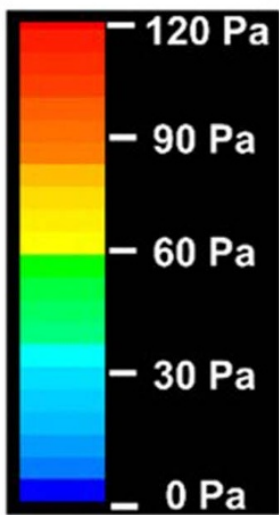

c)

d)
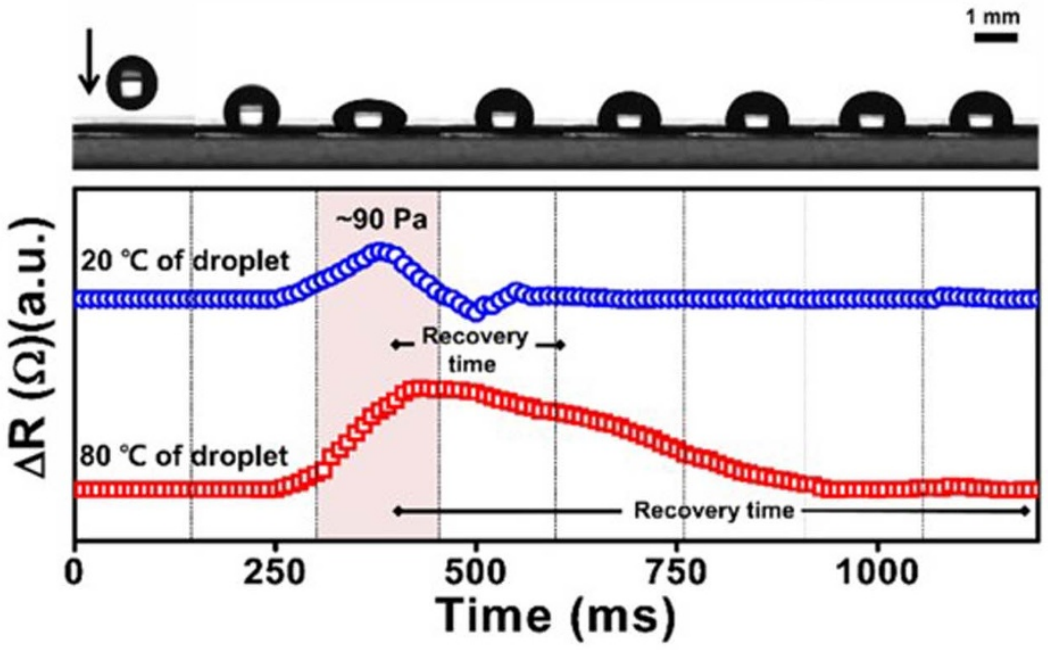

Figure 8 The relationship between external pressure and the temperature of the object placed on the device to induce a pressure response. (a) The change in the resistance and recovery time. (b) Pseudocolor plots showing the applied pressure on the PVDF/ZnO nanorod film. (c) The response to impact of a droplet with an unknown temperature. (d) The recovery time shows that the temperatures of the droplets were $20^{\circ} \mathrm{C}$ and $80^{\circ} \mathrm{C}$, and the pressure was $90 \mathrm{~Pa}$.

minimum level required for artificial skin, could be monitored in real time, and the temperature could be inferred from the recovery time of the signal. This favorable performance of the e-skin represents a significant advance over the current state of the art, and has potential applications in biorobotic fields.

\section{Methods}

Materials. Graphite was purchased from Sigma-Aldrich. The graphene electrodes were printed using a commercially available office inkjet printer (Canon Pixima Ip1300). The polyethylene terephthalate (PET) film, polyvinylidene fluoride (PVDF) pellets (with a molecular weight of 275,000 , determined via gel permeation chromatography), zinc acetate dihydrate, zinc nitrate hexahydrate, hexamethylenetertramine and ethanol were obtained from Sigma-Aldrich. PVDF film was obtained from the Fils Corporation (South Korea).

Synthesis of GO dispersion for inkjet printing. GO was synthesized from natural graphite via the modified Hummers and Offeman method, as described by Kovtyukhova and colleagues. Synthesized purified GO suspensions were exfoliated in water by sonication for $1.5 \mathrm{~h}$ to avoid blocking the nozzle. The obtained brown dispersion was then washed for 4 cycles of centrifugation at 5,000 rpm to remove any unexfoliated GO.

Modification of the inkjet printer. The ink cartridge (printer head) was disassembled and washed several times using ethanol and distilled water after removing all the inks. The exfoliated GO was dispersed distilled water at $0.1 \mathrm{wt} \%$, and was injected into the modified cartridge. The sealed ink cartridge was then placed in the printer body for use in further experiments.

Preparation of ZnO. Zinc acetate was dissolved in $100 \mathrm{~mL}$ of ethanol, spin-coated onto the rGO-treated PET film, placed in an oven at $100^{\circ} \mathrm{C}$, and dried for $30 \mathrm{~min}$ to create a seed layer for subsequent growth of the $\mathrm{ZnO}$ nanostructures. A $500-\mathrm{mL}$ solution of $0.05 \mathrm{M}$ hexamethylenetetramin and $0.05 \mathrm{M}$ zinc nitrate hexahydrate and de-ionized water was then coated onto the substrate, and the $\mathrm{ZnO}$

nanostructures were grown at $90^{\circ} \mathrm{C}$ for 3-7 h to control the morphology of $\mathrm{ZnO}$ nanostructures.

Preparation of the PVDF thin film. PVDF was dissolved in a 1:1 mixture of DMF and acetone. The solution was stirred at $60^{\circ} \mathrm{C}$ for $12 \mathrm{~h}$ and dropped onto the substrate containing the $\mathrm{ZnO}$ nanostructures. Following spin-coating at $1200 \mathrm{rpm}$ for $150 \mathrm{~s}$, the film was poled under an electric field of $300 \mathrm{kV} / \mathrm{cm}$ at $90^{\circ} \mathrm{C}$.

Characterization. HR-TEM images were captured using a JEOL JEM-3010. Fieldemission scanning electron microscopy (FE-SEM) (JEOL JSM-6700F, Japan) and a FTIR spectroscopy (Bomem MB 100, USA) were also used. Atomic force microscopy (AFM) (Nanoscope IIIa, Digital instruments, USA) was used to image the surface topography. AFM measurements were carried out in tapping mode with silicon tips at a resonance frequency of $320 \mathrm{kHz}$. The electrical resistance was measured using a Keithley 2400 source meter at $25^{\circ} \mathrm{C}$ using a four-probe method. Raman spectra were recorded using a LabRAM HR (Horiba, Japan) with 1064-nm laser excitation. For the analysis of detailed force responses, a computer-based user interface and a micro pressure sensor (FT-S270, Nano Science Instrument) with nanoscale-controlled stage by piezoelectric stepping positioner (SLC-1730, Nano Science Instrument) were used to apply an external pressure. Moreover, direction of stepper to apply pressure was vertically loaded and unloaded in order to avoid distortion output signals by torsion or sheer stress.

1. Pang, C. et al. Flexible and highly sensitive strain-gauge sensor using reversible interlocking of nanofibres. Nature Mater. 11, 795-901 (2012).

2. Pecora, A., Maiolo, L., Maita, F. \& Minotti, A. Flexible PVDF-TrFE pyroelectric sensor driven by polysilicon thin film transistor fabricated on ultra-thin polyimide substrate. Sensor Actuat. A-Phys. 185, 39-43 (2012). 
3. Hammock, M. L. et al. 25th Anniversary Article: The Evolution of Electronic Skin (E-Skin): A Brief History, Design Considerations, and Recent Progress. Adv. Mater. 25, 5997-6038 (2013).

4. Lee, J. S., Shin, K.-Y., Kim, C. \& Jang, J. Enhanced frequency response of a highly transparent PVDF-graphene based thin film acoustic actuator. Chem. Commum. 49, 11047-11049 (2013).

5. Kim, D.-H. et al Epidermal Electronics. Science. 333, 838-843 (2011).

6. Moon, H. G. et al. Self-activated ultrahigh chemosensitivity of oxide thin film nanostructures for transparent sensors. Sci. Rep. 2, 588 (2012).

7. Mao, S. et al. Direct Growth of Vertically-oriented Graphene for Field-Effect Transistor Biosensor. Sci. Rep. 3, 1696 (2013).

8. Yamada,T. et al. A stretchable carbon nanotube strain sensor for human-motion detection. Nature Nanotech. 27, 296-301 (2011).

9. Gómez-Martínez, R. et al. Silicon chips detect intracellular pressure changes in living cells. Nature Nanotech. 8, 517-521 (2013).

10. Bi, H. et al. Ultrahigh humidity sensitivity of graphene oxide. Sci. Rep. 3, 2714 (2013).

11. Ehre, D., Lyahovitskaya,V., Tagantsev, A. \& Lubomirsky, I. Amorphous Piezoand Pyroelectric Phases of BaZrO3 and SrTiO3. Adv. Mater. 19, 1515-1517 (2007).

12. Yeo, W.-H. et al. Multifunctional Epidermal Electronics Printed Directly Onto the Skin. Adv. Mater. 25, 2773-2778 (2013).

13. Mönch, I. et al. Rolled-Up Magnetic Sensor: Nanomembrane Architecture for InFlow Detection of Magnetic Objects. ACS Nano. 9, 7436-7442 (2011).

14. Calvert, P. Piezoelectric Polyvinylidene Fluoride. Nature. 256, 694 (1975).

15. Lovinger, A. J. Ferroelectric Polymers. Science. 220, 1115-1121 (1983).

16. Kim, J.-H., Beak, J. \& Shiv Halasyamani, P. $\left(\mathrm{NH}_{4}\right)_{2} \mathrm{Te}_{2} \mathrm{WO}_{8}$ : A New Polar Oxide with Second-Harmonic Generating, Ferroelectric, and Pyroelectric Properties. Chem. Mater. 19, 5637-5641 (2007).

17. Shin, K.-Y., Hong, J.-Y. \& Jang, J. Micropatterning of Graphene Sheets by Inkjet Printing and Its Wideband Dipole-Antenna Application. Adv. Mater. 23, 21132118 (2011)

18. Tieke, B. Langmuir-Blodgett Films for Electronic Applications. Adv. Mater. 2, 222-231 (1990).

19. Naciri, J. D. et al. Synthesis and Pyroelectric Properties of Novel Ferroelectric Organosiloxane Liquid Crystalline Materials. Chem. Mater. 14, 5134-5139 (2002).

20. Ma, J., Hu, J., Li, Z. \& Nan, C.-W. Recent Progress in Multiferroic Magnetoelectric Composites: from Bulk to Thin Films. Adv. Mater. 23, 1062-1087 (2011).

21. Cha, S. et al. Porous PVDF As Effective Sonic Wave Driven Nanogenerators. Nano Lett. 11, 5142-5147 (2011).

22. Yang, Y. et al. Flexible Hybrid Energy Cell for Simultaneously Harvesting Thermal, Mechanical, and Solar Energies. ACS Nano. 7, 785-790 (2013).

23. Hu, W. J. et al. Universal Ferroelectric Switching Dynamics of Vinylidene Fluoride-trifluoroethylene Copolymer Films. Sci. Rep. 4, 4772 (2014).

24. Hansen, B. J., Liu, Y., Yang, R. \& Wang, Z. L. Hybrid Nanogenerator for Concurrently Harvesting Biomechanical and Biochemical Energy. ACS Nano. 7, 3647-3652 (2010).

25. Omar, K., Ooi, J. \& Hassin, M. M. Investigation on Dielectric Constant of Zinc Oxide. Modern Appl. Sci. 3, 110-116 (2009).

26. Ito, D., Jespersen, M. L. \& Hutchison, J. E. Selective Growth of Vertical ZnO Nanowire Arrays Using Chemically Anchored Gold Nanoparticles. ACS Nano. 2, 2001-2006 (2008).

27. Shin, K.-Y., Hong, J.-Y. \& Jang, J. Flexible and transparent graphene films as acoustic actuator electrodes using inkjet printing. Chem. Commum. 47, $8527-8529$ (2011).

28. Choi, S. W., Kim, J. R., Ahn, Y. R., Jo, S. M. \& Cairns, E. J. Characterization of Electrospun PVdF Fiber-Based Polymer Electrolytes. Chem. Mater. 19, 104-115 (2007).

29. Kim, K.-H., Lee, K. Y., Seo, J.-S., Kumar, B. \& Kim, S.-W. Paper-Based Piezoelectric Nanogenerators with High Thermal Stability. Small. 7, 2577-2580 (2011).

30. Hong, J.-Y. \& Jang, J. Micropatterning of graphene sheets: recent advances in techniques and applications. J. Mater. Chem. 22, 8179-8191 (2012).

31. Shin, K.-Y. \& Jang, J. Highly conductive, flexible and scalable graphene hybrid thin films with controlled domain size as transparent electrodes. Chem. Соттит. 50, 6645-6648 (2014).
32. Panigrahy, B., Aslam, M. \& Bahadur, D. Effect of Fe doping concentration on optical and magnetic properties of $\mathrm{ZnO}$ nanorods. Nanotechnology. 23, 115601 (2012).

33. Baruah, S. \& Dutta, J. Hydrothermal growth of $\mathrm{ZnO}$ nanostructures. Sci. Technol. Adv. Mater. 10, 13001 (2009).

34. Chang, D. H. \& Yoon, Y. S. Pyroelectric Properties of the B-Polyvilnylidene Fluoride (PVDF) Thin Film Prepared by Vacuum Deposition with Electric Field Application. Jpn. J. Appl. Phys. 41, 7234-7238 (2002).

35. Lee, S., Yoon, C.-M. Hong, J.-Y. \& Jang, J. Enhanced electrorheological performance of a graphene oxide-wrapped silica rod with a high aspect ratio. J. Mater. Chem. C. 2, 6010-6016 (2014).

36. Hong, J.-Y., Lee, E. \& Jang, J. Electro-responsive and dielectric characteristics of graphene sheets decorated with TiO2 nanorods. J. Mater. Chem. A. 1, 117-121 (2013).

37. Segev-Bar, M., Landman, A., Nir-Shapira, M., Shuster, G. \& Haick, H. Tunable Touch Sensor and Combined Sensing Platform: Toward Nanoparticle-based Electronic Skin. ACS Appl. Mater. Interfaces. 5, 5531-5541 (2013).

38. Tee, B. C-K., Wang, C., Allen, R. \& Bao, Z. An electrically and mechanically selfhealing composite with pressure- and flexion-sensitive properties for electronic skin applications. Nature Nanotechnology. 7, 825-832 (2012).

39. Van Boven, R. W. \& Johnson, K. O. The limit of tactile spatial resolution in humans. Neurology. 44, 2361 (1994).

40. Dellon, E. S., Mourey, R., Dellon, Lee, A. \& Dellon. Human Pressure Perception Values for Constant and Moving One- and Two-Point Discrimination. Plastic \& Reconstructive Surgery. 90, 112-117 (1992).

41. Lang, S. B. et al. Ferroelectric Polarization in Nanocrystalline Hydroxyapatite Thin Films on Silicon. Sci. Rep. 3, 2215 (2013).

42. Jiang, Z. Y., Zheng, G. P., Han, Z., Liu, Y. Z. \& Yang, J. H. Enhanced ferroelectric and pyroelectric properties of poly(vinylidene fluoride) with addition of graphene oxides. J. Appl. Phys. 115, 204101 (2014).

43. Chang, H. H. S., Whatmore, R. W. \& Huang, Z. Pyroelectric effect enhancement in laminate composites under short circuit condition. J. Appl. Phys. 106, 114110 (2009).

44. Qiu, X. Patterned piezo-, pyro-, and ferroelectricity of poled polymer electrets. J. Appl. Phys. 108, 11101 (2010).

\section{Acknowledgments}

This research was supported by the National Research Foundation of Korea (NRF) grant funded by the Korean government (MEST) (Grant no. 2011-0017125).

\section{Author contributions}

J.S.L and K.Y.S. contributed equally to this work; J.S.L., K.Y.S., O.J.C. and J.H.K. designed and performed the experiments, and authored the manuscript; J.S.L. and K.Y.S. contributed to data collection and theoretical analysis; J.J. planned and supervised the project; All authors edited the manuscript.

\section{Additional information}

Supplementary information accompanies this paper at http://www.nature.com/ scientificreports

Competing financial interests: The authors declare no competing financial interests. How to cite this article: Lee, J.S., Shin, K.-Y., Cheong, O.J., Kim, J.H. \& Jang, J. Highly Sensitive and Multifunctional Tactile Sensor Using Free-standing ZnO/PVDF Thin Film with Graphene Electrodes for Pressure and Temperature Monitoring. Sci. Rep. 5, 7887; DOI:10.1038/srep07887 (2015)

This work is licensed under a Creative Commons Attribution 4.0 International License. The images or other third party material in this article are included in the article's Creative Commons license, unless indicated otherwise in the credit line; if the material is not included under the Creative Commons license, users will need to obtain permission from the license holder in order to reproduce the material. To view a copy of this license, visit http://creativecommons.org/licenses/by/4.0/ 